Article

\title{
Dynamic Response of a Permanent Magnet Synchronous Generator to a Wind Gust
}

\author{
Nicolae Herisanu ${ }^{1,2, *}$, Vasile Marinca ${ }^{1,2}$, Gheorghe Madescu ${ }^{2}$ and Florin Dragan ${ }^{1}$ \\ 1 University Politehnica Timisoara, Timisoara 300006, Romania; vasile.marinca@upt.ro (V.M.); \\ florin.dragan@upt.ro (F.D.) \\ 2 Romanian Academy, Centre for Advanced Technical Research, Timisoara 300223, Romania; \\ gmadescu@d109lin.upt.ro \\ * Correspondence: nicolae.herisanu@upt.ro; Tel.: +40-256-403629
}

Received: 5 February 2019; Accepted: 5 March 2019; Published: 9 March 2019

check for updates

\begin{abstract}
In this paper, a new analytical approach is proposed to investigate the electrical and mechanical behavior of a low-power permanent magnet synchronous generator (PMSG) in the presence of a wind gust. The proposed model for the wind gust and wind power system was analytically investigated using the optimal auxiliary functions method (OAFM), which has proven to be a reliable tool. The reaction of the system to a wind gust was explicitly obtained, which is useful for stability analysis, protection issues, and risk assessment concerning the PMSG. A substantial reduction of computations in analytically analysing a complicated dynamical system is ensured by this new approach, through some auxiliary functions and convergence-control parameters.
\end{abstract}

Keywords: permanent magnet synchronous generator; wind gust; optimal auxiliary functions method

\section{Introduction}

Wind turbine dynamics recently became a subject of great interest for scientists [1,2], with the increasing importance of renewable, green energy harvesting technologies and their installations.

It is a known reality that both mechanical and electrical loads are greatly influenced by the action of the wind speed and its variations, which simultaneously affect the tower/nacelle and blade system, and also the electrical parameters or the mechanical behavior of the stator/rotor system. It has been shown that random wind fluctuations can excite significant variations in wind turbine torque as well as in generated electric power [3,4]. Other works reveal the dynamic response of permanent magnet synchronous generators (PMSGs) to specific wind speed profiles in order to assess both the electrical and mechanical stress of different components of the wind power station [5] or propose some modeling procedures for wind speed simulation, which are needed in the investigation of wind power systems [6].

The jumps in wind speed represented by wind gusts are often present in real operating conditions and can produce mechanical and electrical shocks, which can lead to damages from both mechanical and electrical points of view. From a mechanical point of view, the wind gusts generate torque pulsations in the drive train, and consequently an additional mechanical stress occurs. Therefore, it is very important to know how such wind variations affect the mechanical and electrical performance of the wind turbine in order to develop an efficient tool to evaluate and control the whole phenomenon. The influence of wind gusts on various wind power systems is a topic of increased interest for engineers. Giaourakis and Safacas [7] presented a quantitative and qualitative behavior analysis of a wind energy conversion system under a wind gust and converter faults, which was carried out via simulation. Borowy and Salameh [8] studied the dynamic response of a stand-alone wind energy conversion 
system with battery energy storage to a wind gust. Bystryk and Sullivan [9] analyzed control strategies for a small-scale wind turbine in intermittent wind gusts using a computer model.

Generally, such developments need some reliable models, which must obey the theory of electrical machines [10-13]. Once such a complex model is achieved, the solving process can be managed by various analytical and/or numerical approaches. Usually, perturbation methods or asymptotic approaches are used to solve these kinds of non-linear problems [14,15]. Moreover, recently, some new and emerging approaches were proposed to handle systems with strong nonlinearities or consider large parameters or large domains of definition. Among them may be mentioned the perturbation-iteration method (PIM), a technique which combines features of the perturbative and iterative approaches presented in [16], where it was found that it performs better than the well-known variational iteration method (VIM), since a few iterations are actually sufficient to obtain satisfactory results. Similar techniques are applied in Reference [17] for the Van der Pol oscillator and in Reference [18] for different types of non-linear equations, where the perturbation-iteration technique is compared with the early-stage version of the optimal homotopy asymptotic method. Other relatively new approaches are the homotopy analysis method (HAM) [19] and the homotopy perturbation method (HPM) [20], which are two homotopic approaches that have been successfully applied in solving numerous non-linear problems in science and engineering, for both of them the accuracy of the results increasing as the number of iterations increases, such that higher-order approximations provide more accurate results. Two other improved homotopic approaches are the optimal homotopy perturbation method (OHPM) [21] and the optimal homotopy asymptotic method (OHAM) [22,23], which are endowed with a rigorous and reliable procedure for convergence control, which lead to an increase in accuracy at a reduced number of iterations. Finally, among these new and emerging approaches intended for solving non-linear problems can be mentioned the modified differential transform method (MDTM) [24], which is used mainly in solving boundary values problems.

In this paper, the electrical and mechanical reaction of a low-power PMSG in the presence of a wind gust is investigated using a new approach, namely, the optimal auxiliary functions method (OAFM). Explicit analytical solutions are developed and analyzed in order to emphasize the effect of the wind gust from both mechanical and electrical points of view. Generally, such analytical approaches are very useful to further develop an efficient tool for protection issues and risk assessments concerning the PMSG.

\section{Dynamic Model of the Wind-Power System}

A wind-power system consists of different subsystems which should be separately modeled in view of the simulation of the whole system. Such subsystems are studied in this work. For this purpose, using the classical D-Q equivalent circuit models, the equations of the smooth-air-gap synchronous machine in the rotor reference frame can be written in the form [10-13]:

$$
\begin{aligned}
& u_{D}=R_{G} i_{D}+\frac{d \psi_{D}}{d t}-\omega_{E} \psi_{Q} \\
& u_{Q}=R_{G} i_{Q}+\frac{d \psi_{Q}}{d t}+\omega_{E} \psi_{D} \\
& \psi_{D}=L_{G} i_{D}+\psi_{P M} \\
& \psi_{Q}=L_{G} i_{Q}
\end{aligned}
$$

where $u_{D}, u_{Q}, i_{D}$, and $i_{Q}$ are instantaneous values of $D$ and $Q$ axis stator voltage components and stator current components, respectively; $R_{G}$ and $L_{G}$ are electrical resistance and synchronous inductance of the generator phase windings; $\psi_{D}$ and $\psi_{Q}$ are instantaneous values of $\mathrm{D}$ and $\mathrm{Q}$ axis stator flux components; $\psi_{P M}$ is the permanent magnet flux; and $\omega_{E}$ is the electrical angular speed. The mechanical angular speed of the turbine-generator system is $\Omega_{M}=\omega_{E} P_{1}^{-1}$, where $P_{1}$ is the number of pole pairs of the generator. 
Since the system (1) has been written for motoring, the PMSG output voltages are:

$$
\begin{aligned}
& u_{D}=-R_{L} i_{D} \\
& u_{Q}=-R_{L} i_{Q}
\end{aligned}
$$

where $R_{L}$ is the electrical resistance of the external load connected to the output of the generator.

To investigate the electromechanical dynamic behavior, the motion equation of the generator can be written as [25]:

$$
\frac{J_{M}}{P_{1}} \frac{d \omega_{E}}{d t}=T_{M}+\frac{3}{2} P_{1} \psi_{P M} i_{Q}
$$

where $J_{M}$ is the axial moment of inertia (total inertia) and $T_{M}$ is the mechanical torque of the wind turbine, which can be expressed as $[8,26]$ :

$$
T_{M}=\frac{1}{2} \rho \pi r^{3} v^{2} C_{t}\left(\lambda_{r}\right), \quad \lambda_{r}=\frac{r \omega_{E}}{P_{1} v},
$$

where $\rho$ is the air density, $r$ is the turbine radius, $v$ is the wind speed, $\lambda_{r}$ is the tip-speed ratio, and $C_{t}$ is the torque coefficient provided by the turbine manufacturer:

$$
C_{t}\left(\lambda_{r}\right)=0.125+0.2092 \lambda_{r}-0.1209 \lambda_{r}^{2.5} .
$$

Using the values $\rho=1.225 \mathrm{Kg} \cdot \mathrm{m}^{-3}, P_{1}=16$, and $r=2.5 \mathrm{~m}$, the final form of the torque becomes:

$$
T_{M}=T_{M}\left(v, \omega_{E}\right)=3.758252931 v^{2}+0.982783141 v \omega_{E}-\frac{0.035079416}{\sqrt{v}} \omega_{E}^{2.5} .
$$

The model of the wind speed is described by a variable function with respect to time, which simulates the manner in which the "wind profile" is altered. In this work it is considered that the wind profile contains two components:

$$
v(t)=v_{m}+v_{G}(t),
$$

where $v_{m}$ is the mean wind speed or the base wind velocity, which is a constant, and $v_{G}$ is the gust wind component. The base wind velocity $v_{m}$ is considered only in the case in which the generator is active, and the gust wind velocity component can be considered as the usual (1-cosine) gust [27]. Therefore, one can consider:

$$
v_{m}=\text { const., } \quad v_{G}(t)=\frac{A}{2}\left(1-\cos \frac{2 \pi}{T_{G}} t\right),
$$

where $A$ is the gust peak and $T_{G}$ is the gust period.

The considered PMSG, which is a component of the wind power station, had the following characteristics in the steady-state regime: $R_{G}=0.9 \Omega, L_{G}=0.03 \mathrm{H}, \psi_{P M}=1.42 \mathrm{~Wb}, P_{1}=16$ pole pairs, $J_{M}=4.75 \mathrm{kgm}^{2}$.

It was also considered that the nominal speed of rotation in the steady-state nominal regime was $n_{N}=70 \mathrm{rpm}$, which means that $\Omega_{N}=7.330352856 \mathrm{rad} \cdot \mathrm{s}^{-1}$ or $\omega_{N}=117.28 \mathrm{rad} \cdot \mathrm{s}^{-1}$. In all cases, the index $N$ denotes the nominal values (or rated values). The motion of the wind turbine is determined by the constant wind speed $v_{N}$.

By means of the above values, from Equation (6) one retrieves $T_{M N}=684.192163461 \mathrm{Nm}$ and from Equation (3) for the steady-state regime one can obtain $i_{Q N}=-20.158204693 \mathrm{~A}$. Taking this last value into account and using Equations (1) and (2), it follows that $i_{D N}=-11.118492391 \mathrm{~A} ; R_{L N}=$ $5.479285888 \Omega ; u_{D N}=60.9213984503 \mathrm{~V} ; u_{\mathrm{QN}}=110.452566480 \mathrm{~V} ; \sqrt{2} I_{N}=\sqrt{i_{D N}^{2}+i_{Q N}^{2}}=23.02116616$ $\mathrm{A} ; \sqrt{2} U_{N}=\sqrt{u_{D N}^{2}+u_{Q N}^{2}}=126.139550551 \mathrm{~V}$. 
It follows that in the nominal point of working, the wind turbine would develop the mechanical power $P_{1 N}=T_{M N} \Omega_{N} \cong 5015 \mathrm{~W}$ and the electric generator would develop the electrical power $P_{2 N}=3 U_{N} I_{N} \cong 4355.82 \mathrm{~W}$.

For investigation purposes, it is often convenient to express the generator's parameters, variables, and governing equations using dimensionless quantities. In this respect, these terms are divided by base quantities. The following set of base quantities is widely used in such investigations $[10,25,28]$ : the base voltage $U_{B}$ (peak stator phase nominal voltage $U_{B}=\sqrt{2} U_{N}$ ); the base current $I_{B}$ (peak stator phase nominal current $I_{B}=\sqrt{2} I_{N}$ ); the base power $S_{B}$ (nominal apparent power $S_{B}=3 U_{N} I_{N}$ ); the base angular speed $\omega_{B}$ (nominal electrical angular speed $\omega_{B}=\omega_{N}$ ). Based on this restricted set, one can obtain additional quantities, such as: the base torque $T_{B}=P_{1} S_{B} / \omega_{B}$; the base flux linkage $\psi_{B}=U_{B} / \omega_{B}$; the base impedance $Z_{B}=U_{B} / I_{B}$; and the base time $t_{B}=1 / \omega_{B}$.

By means of the following notations:

$$
\begin{aligned}
& u_{d}=\frac{u_{D}}{U_{B}} ; u_{q}=\frac{u_{Q}}{U_{B}} ; i_{d}=\frac{i_{D}}{I_{B}} ; i_{q}=\frac{i_{Q}}{I_{B}} ; \psi_{d}=\frac{\psi_{D}}{\psi_{B}} ; \psi_{q}=\frac{\psi_{Q}}{\psi_{B}} ; \psi_{p m}=\frac{\psi_{P M}}{\psi_{B}} ; \\
& \omega=\frac{\omega_{E}}{\omega_{B}} ; r_{l}=\frac{R_{L}}{Z_{B}} ; r_{g}=\frac{R_{G}}{Z_{B}} ; x_{g}=\frac{\omega_{B} L_{G}}{Z_{B}} ; T_{m}=\frac{T_{M}}{T_{B}} ; k=\frac{J_{M} \omega_{B}^{3}}{3 P_{1}^{2} U_{N} I_{N}} ; \tau=\frac{t}{t_{B}}=\omega_{B} t
\end{aligned}
$$

the governing Equations (1) may be written as:

$$
\begin{aligned}
& \frac{d i_{d}}{d \tau}-\omega i_{q}+\frac{r_{l}+r_{g}}{x_{g}} i_{d}=0 \\
& \frac{d i_{q}}{d \tau}+\omega i_{d}+\frac{r_{l}+r_{g}}{x_{g}} i_{q}+\omega \frac{\psi_{p m}}{x_{g}}=0 \\
& k \frac{d \omega}{d \tau}-T_{m}-\psi_{p m} i_{q}=0
\end{aligned}
$$

The initial conditions for Equations (10) are obtained from the working condition of a considered regime, established before the gust occurs, which is considered as the steady-state regime, characterized by a constant angular speed $\omega_{0}$, at a constant wind speed of $v_{m}=10 \mathrm{~m} \cdot \mathrm{s}^{-1}$ and an external electrical load $r_{l}=0.45248$. Taking these data into account, considering the steady-state regime in Equation (10), one gets the following initial conditions:

$$
\begin{aligned}
& i_{d}(0)=-0.438786995 \\
& i_{q}(0)=-0.843879596 \\
& \omega(0)=0.499239911
\end{aligned}
$$

In this way, the governing equations become:

$$
\begin{aligned}
& \frac{d i_{d}}{d \tau}-\omega i_{q}+0.960143255 i_{d}=0 \\
& \frac{d i_{q}}{d \tau}+\omega i_{d}+0.960143255 i_{q}+2.061756973 \omega=0 \\
& \frac{d \omega}{d \tau}-0.168386689 i_{q}-0.000808844 v^{2}-0.024807429 v \omega+\frac{1.124718044}{\sqrt{v}} \omega^{2.5}=0
\end{aligned}
$$

By means of the change of the variable $\tau=\omega_{B} t$ and considering $A=6, v_{m}=10 \mathrm{~m} \cdot \mathrm{s}^{-1}$, and $T_{G}=$ 12 in Equation (8), Equation (7) can be expressed as:

$$
v(\tau)=10+3\left(1-\cos \frac{2 \pi}{\lambda} \tau\right) .
$$

The dynamical system described above is analyzed in the following using a new solution procedure, namely the optimal auxiliary functions method.

\section{Basic Ideas of the Optimal Auxiliary Functions Method}

The most general form of a non-linear differential equation can be expressed as [29]:

$$
L[f(\tau)]+N[f(\tau)]=0,
$$


where $L$ is a linear operator, $N$ is a non-linear operator, and $f(\tau)$ is an unknown function. The initial/boundary conditions are:

$$
B\left(f(\tau), \frac{d f(\tau)}{d \tau}\right)=0 .
$$

In the following, it is assumed that Equations (14) and (15) have an approximate solution in a two-component form:

$$
\widetilde{f}\left(\tau, C_{i}\right)=f_{0}(\tau)+f_{1}\left(\tau, C_{i}\right), \quad i=1,2, \ldots, s,
$$

where the initial approximation $f_{0}(\tau)$ and the first approximation $f_{1}\left(\tau, C_{i}\right)$ will be determined as follows. Substituting Equation (16) into Equation (14), it is obtained that

$$
L\left(f_{0}(\tau)\right)+L\left(f_{1}\left(\tau, C_{i}\right)\right)+N\left[f_{0}(\tau)+f_{1}\left(\tau, C_{i}\right)\right]=0 .
$$

The initial approximation $f_{0}(\tau)$ is determined from the linear equation

$$
\begin{gathered}
L\left[f_{0}(\tau)\right]=0 \\
B\left(f_{0}(\tau), \frac{d f_{0}(\tau)}{d \tau}\right)=0
\end{gathered}
$$

and the first approximation $f_{1}\left(\tau, C_{i}\right)$ from the remaining equation

$$
\begin{aligned}
& L\left[f_{1}\left(\tau, C_{i}\right)\right]+N\left[f_{0}(\tau)+f_{1}\left(\tau, C_{i}\right)\right]=0 \\
& B\left(f_{1}(\tau), \frac{d f_{1}(\tau)}{d \tau}\right)=0
\end{aligned}
$$

In general, Equation (19) is a non-linear differential equation which is often very difficult to solve. Now, the non-linear term from Equation (19) is expanded in the form:

$$
N\left[f_{0}(\tau)+f_{1}\left(\tau, C_{i}\right)\right]=N\left[f_{0}(\tau)\right]+\sum_{k=1}^{\infty} \frac{f_{1}^{k}(\tau)}{k !} N^{(k)}\left[f_{0}(\tau)\right]
$$

In order to avoid the difficulties that appear in solving the non-linear differential Equation (19) and to accelerate the rapid convergence of the first approximation-and implicitly of the approximate solution $\widetilde{f}(\tau)$-instead of the last term arising in Equation (19), another expression is proposed, such that Equation (19) can be written as

$$
\begin{aligned}
& L\left[f_{1}\left(\tau, C_{i}\right)\right]+A_{1}\left(f_{0}(\tau), C_{i}\right) N\left[f_{0}(\tau)\right]+A_{2}\left(f_{0}(\tau), C_{j}\right)=0 \\
& B\left(f_{1}\left(\tau, C_{i}\right), \frac{d f_{1}\left(\tau, C_{i}\right)}{d \tau}\right)=0
\end{aligned}
$$

where $A_{1}$ and $A_{2}$ are two arbitrary auxiliary functions depending on the initial approximation $f_{0}(\tau)$ and several unknown parameters $C_{i}$ and $C_{j}, i=1,2, \ldots, p, j=p+1, p+2, \ldots, s$. The auxiliary functions $A_{1}$ and $A_{2}$-called optimal auxiliary functions - are not unique, and are of the same form as $f_{0}(\tau)$ or of the form of $N\left[f_{0}(\tau)\right]$, or combinations of the forms of $f_{0}(\tau)$ and $N\left[f_{0}(\tau)\right]$. As illustrative examples, if $f_{0}(\tau)$ or $N\left[f_{0}(\tau)\right]$ are polynomial functions, then $A_{1}\left[f_{0}(\tau), C_{i}\right]$ and $A_{2}\left[f_{0}(\tau), C_{j}\right]$ are sums of polynomial functions; if $f_{0}(\tau)$ or $N\left[f_{0}(\tau)\right]$ contain exponential functions, then $A_{1}$ and $A_{2}$ would be sums of exponential functions; if $f_{0}(\tau)$ or $N\left[f_{0}(\tau)\right]$ are trigonometric functions, then $A_{1}$ and $A_{2}$ would be sums of trigonometric functions, and so on. If, in a special case, $N\left[f_{0}(\tau)\right]=0$, then it is clear that $f_{0}(\tau)$ is an exact solution of Equations (11) and (12).

The unknown parameters $C_{i}$ and $C_{j}$ can be optimally identified using different methods. Among them, a reliable method would be minimizing the square residual error by

$$
J\left(C_{i}, C_{j}\right)=\int_{a}^{b} R^{2}\left(\tau, C_{i}, C_{j}\right) d \tau,
$$


where $R\left(\tau, C_{i}, C_{j}\right)=L\left[\widetilde{f}\left(\tau, C_{i}, C_{j}\right)\right]+N\left[\widetilde{f}\left(\tau, C_{i}, C_{j}\right)\right], i=1,2, \ldots p ; j=p+1, p+2, \ldots, s$. Then, the conditions of minimization would be

$$
\frac{\partial J}{\partial C_{1}}=\frac{\partial J}{\partial C_{2}}=\ldots=\frac{\partial J}{\partial C s}=0 .
$$

Alternatively, one can use other approaches such as the Ritz method, the collocation method, the Galerkin method, and so on. Finally, by this novel approach, after the identification of the optimal convergence-control parameters, the approximate solution to (16) is determined well. Our procedure proves to be a powerful tool for solving non-linear problems not depending on small or large parameters. It should be emphasized that our method contains the optimal auxiliary functions $A_{1}$ and $A_{2}$, which provides us with a simple way to adjust and control the convergence of the approximate solutions after only the first iteration.

\section{Approximate Solution of the Dynamic Model of the Wind-Power System}

In the following, the above-described procedure to obtain an approximate solution for Equations (11) and (12) is applied. The linear operators for System (12) are

$$
L_{1}\left(i_{d}(\tau)\right)=\frac{d i_{d}(\tau)}{d \tau} ; L_{2}\left(i_{q}(\tau)\right)=\frac{d i_{q}(\tau)}{d \tau} ; L_{3}(\omega(\tau))=\frac{d \omega(\tau)}{d \tau} .
$$

The approximate solutions given by Equation (16) in our case can be written as

$$
\begin{array}{ll}
\widetilde{i}_{d}(\tau)=i_{d 0}(\tau)+i_{d 1}\left(\tau, C_{i}\right), & i=1,2, \ldots \\
\widetilde{i}_{q}(\tau)=i_{q 0}(\tau)+i_{q 1}\left(\tau, D_{j}\right), & j=1,2, \ldots \\
\widetilde{\omega}(\tau)=\omega_{0}(\tau)+\omega_{1}\left(\tau, E_{k}\right), & k=1,2, \ldots
\end{array}
$$

The initial approximations $i_{d 0}, i_{q 0}$, and $\omega_{0}$ are determined from Equations (18) and (11), which in this case read:

$$
\begin{array}{ll}
\frac{d i_{d 0}(\tau)}{d \tau}=0 & i_{d 0}(0)=-0.438786995 \\
\frac{d i_{q 0}(\tau)}{d \tau}=0 & i_{q 0}(0)=-0.843879596 \\
\frac{d \omega_{0}(\tau)}{d \tau}=0 & \omega_{0}(0)=0.499239911
\end{array}
$$

The solution of System (26) is

$$
\begin{aligned}
& i_{d 0}(\tau)=-0.438786995 \\
& i_{q 0}(\tau)=-0.843879596 \\
& \omega_{0}(\tau)=0.499239911
\end{aligned}
$$

The non-linear operators for the system (12) are

$$
\begin{gathered}
N_{1}\left(i_{d}, i_{q}, \omega\right)=-\omega i_{q}+0.960143255 i_{d} \\
N_{2}\left(i_{d}, i_{q}, \omega\right)=\omega i_{d}+0.960143255 i_{q}+2.061756973 \omega \\
N_{3}\left(i_{d}, i_{q}, \omega\right)=-0.168386689 i_{q}-0.000808844 v^{2}-0.024807429 v \omega+\frac{1.124718044}{\sqrt{v}} \omega^{2.5}
\end{gathered}
$$

where the wind velocity $v$ is given by Equation (13). By substituting Equations (27) into Equations (28), it holds that

$$
\begin{aligned}
& N_{1}\left(i_{d 0}, i_{q 0}, \omega_{0}\right)=0 \\
& N_{2}\left(i_{d 0}, i_{q 0}, \omega_{0}\right)=0 \\
& N_{3}\left(i_{d 0}, i_{q 0}, \omega_{0}\right)=-0.159239505+0.1002444079 \cos \frac{2 \pi \tau}{\lambda}-0.003639798 \cos \frac{4 \pi \tau}{\lambda}+\frac{0.1980691808}{\sqrt{13-3 \cos \frac{2 \pi \tau}{\lambda}}}
\end{aligned}
$$


The linear equations for the first approximation given by Equations (21), in this case are:

$$
\begin{array}{ll}
\frac{d i_{d 1}(\tau)}{d \tau}+A_{2}\left(\tau, C_{i}\right)=0, & i_{d 1}(0)=0 \\
\frac{d i_{q 1}(\tau)}{d \tau}+A_{2}^{*}\left(\tau, D_{j}\right)=0, & i_{q 1}(0)=0 \\
\frac{d \omega_{1}(\tau)}{d \tau}+A_{1}\left(\tau, E_{k}\right) N_{3}\left(i_{d 0}, i_{q 0}, \omega_{0}\right)+A_{2}^{* *}\left(\tau, E_{k}\right)=0, & \omega_{1}(0)=0
\end{array}
$$

Taking Expression (29) and the initial condition from Equation (30) into account, in the following it is considered that

$$
\begin{aligned}
& A_{2}\left(\tau, C_{i}\right)=-\left[C_{1} \cos \frac{\pi \tau}{\lambda}+C_{2} \cos \frac{3 \pi \tau}{\lambda}+C_{3} \cos \frac{5 \pi \tau}{\lambda}+C_{4} \cos \frac{7 \pi \tau}{\lambda}\right] \\
& A_{2}^{*}\left(\tau, D_{i}\right)=-\left[D_{1} \cos \frac{\pi \tau}{\lambda}+D_{2} \cos \frac{3 \pi \tau}{\lambda}+D_{3} \cos \frac{5 \pi \tau}{\lambda}+D_{4} \cos \frac{7 \pi \tau}{\lambda}+D_{5} \cos \frac{9 \pi \tau}{\lambda}\right] \\
& A_{1}\left(\tau, E_{k}\right)=0 \\
& A_{2}^{* *}\left(\tau, E_{k}\right)=-\left[E_{1} \cos \frac{\pi \tau}{\lambda}+E_{2} \cos \frac{3 \pi \tau}{\lambda}+E_{3} \cos \frac{5 \pi \tau}{\lambda}\right]
\end{aligned}
$$

The first approximations are obtained from Equations (30) and (31) under the form:

$$
\begin{aligned}
& i_{d 1}(\tau)=\frac{\lambda}{\pi}\left[C_{1} \sin \frac{\pi \tau}{\lambda}+\frac{1}{3} C_{2} \sin \frac{3 \pi \tau}{\lambda}+\frac{1}{5} C_{3} \sin \frac{5 \pi \tau}{\lambda}+\frac{1}{7} C_{4} \sin \frac{7 \pi \tau}{\lambda}\right] \\
& i_{q 1}(\tau)=\frac{\lambda}{\pi}\left[D_{1} \sin \frac{\pi \tau}{\lambda}+\frac{1}{3} D_{2} \sin \frac{3 \pi \tau}{\lambda}+\frac{1}{5} D_{3} \sin \frac{5 \pi \tau}{\lambda}+\frac{1}{7} D_{4} \sin \frac{7 \pi \tau}{\lambda}+\frac{1}{9} D_{5} \sin \frac{9 \pi \tau}{\lambda}\right] \\
& \omega_{1}(\tau)=\frac{\lambda}{\pi}\left[E_{1} \sin \frac{\pi \tau}{\lambda}+\frac{1}{3} E_{2} \sin \frac{3 \pi \tau}{\lambda}+\frac{1}{5} E_{3} \sin \frac{5 \pi \tau}{\lambda}\right]
\end{aligned}
$$

The approximate solutions of Equations (11) and (12) are obtained from Equations (27), (32), and (25). The optimal values of $C_{i}, D_{j}$, and $E_{k}$ were determined by the collocation method as follows:

$$
\begin{gathered}
C_{1}=-0.001880098936, C_{2}=0.000249576329, C_{3}=0.0001099044306, C_{4}=0.000047008063 \\
D_{1}=-0.000429218279, D_{2}=-0.0001028685602, D_{3}=0.000044633562, D_{4}=0.000029287755, \\
D_{5}=0.000009332777, E_{1}=0.001592362394, E_{2}=-0.000310689633, E_{3}=-0.000065075109
\end{gathered}
$$

The approximate solution (16) for Equations (11) and (12) can be written as follows:

$$
\begin{aligned}
& i_{d}(\tau)=-0.438786995-0.842240332001 \sin \frac{\pi}{\lambda} \tau+0.111804356099 \sin \frac{3 \pi}{\lambda} \tau+ \\
&+0.049234613317 \sin \frac{5 \pi}{\lambda} \tau+0.021058512367 \sin \frac{7 \pi}{\lambda} \tau \\
& i_{q}(\tau)=-0.843879596-0.192279746121 \sin \frac{\pi}{\lambda} \tau-0.046082708017 \sin \frac{3 \pi}{\lambda} \tau+ \\
&+0.019994791658 \sin \frac{5 \pi}{\lambda} \tau+0.013120229226 \sin \frac{7 \pi}{\lambda} \tau+0.004180865769 \sin \frac{9 \pi}{\lambda} \tau \\
& \omega(\tau)=0.499239911+0.713341093867 \sin \frac{\pi}{\lambda} \tau-0.139181686295 \sin \frac{3 \pi}{\lambda} \tau-0.029152126573 \sin \frac{5 \pi}{\lambda} \tau
\end{aligned}
$$

where $\lambda=1407.36$.

Figures 1-3 show the approximate solution of Equations (11) and (12) which, for validation purposes, were compared with numerical solutions obtained using a fourth-order Runge-Kutta method.

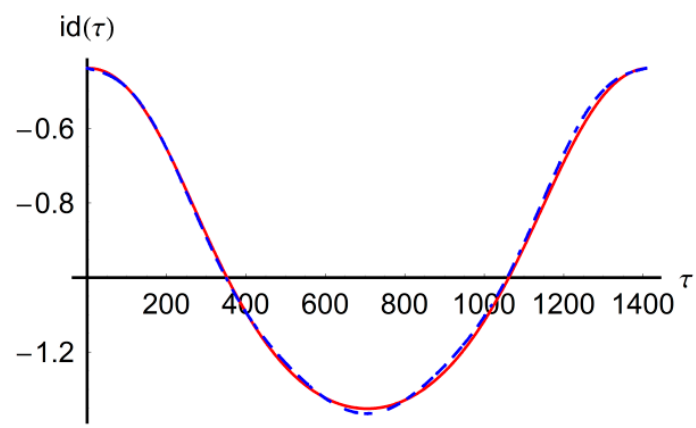

Figure 1. Comparison between the approximate solution and numerical integration results for $i_{d}$ : numerical, analytical solution (33) numerical; analytical. 


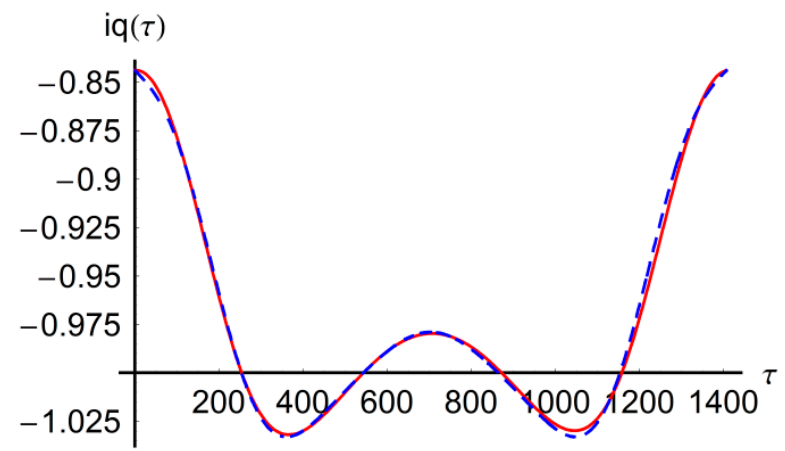

Figure 2. Comparison between the approximate solution and numerical integration results for $i_{q}$ : numerical, analytical solution (34) numerical; analytical.

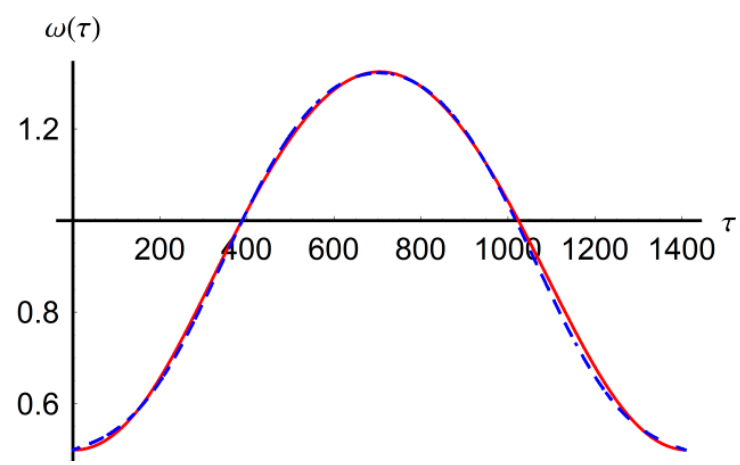

Figure 3. Comparison between the approximate solution and numerical integration results for $\omega$ : numerical, analytical solution (35) numerical; analytical.

The changes occurring in the wind profile at the moment $\tau=0$ generated a non-stationary regime in the system characterized by a variation in the angular speed $\omega$ and also a significant variation in the currents of the generator $i_{d}$ and $i_{q}$. The angular speed increased from the value $\omega=0.5$ to $\omega=1.35$, which is by 2.7 times. Such increases in angular speed under the action of the wind gust could lead to an exceedance of the limit of overload and should be treated with special attention. From Figure 3, one can see that the angular speed of the turbine exceeded the nominal angular speed $(\omega=1)$ by $35 \%$.

These dynamic changes in the operating conditions generated by the wind gust affect the whole mechanical system of the turbine generator, which exhibits mechanical loads emphasized by the mechanical torque, illustrated in Figure 4. In Figure 4 a sudden increase of the torque by almost $20 \%$ can be observed, reaching a maximum at around $\tau=350$. The increasing gradient of the torque was higher than that of the wind speed, which produced substantial mechanical stress in the turbine under the action of the wind gust.

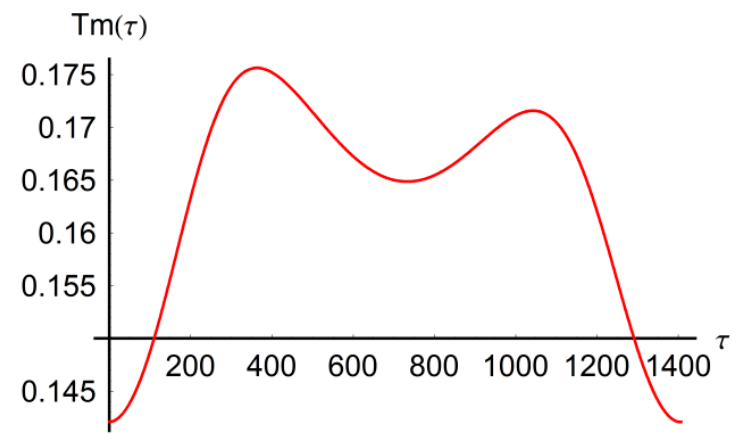

Figure 4. Variation in mechanical torque of the wind turbine during the wind gust. 
One can see from Figure 5 that under the action of the wind gust, the electrical power at the generator had a similar variation as the angular speed, since the electrical voltage is proportional with $\omega$.

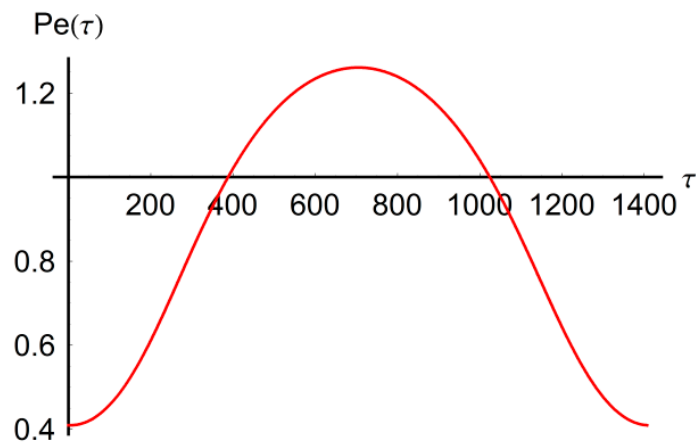

Figure 5. Variation in electrical power during the wind gust $P e=r_{l}\left(i_{d}^{2}+i_{q}^{2}\right)$.

\section{Conclusions}

In the present work, a new technique is proposed to obtain an analytical solution to the dynamic model of a wind-power system. An effective analytic solution is obtained for the governing equations and, as far as we are aware, there are no analytical solutions available in the literature for this particular problem. Comparison with numerical integration results obtained using a fourth-order Runge-Kutta method revealed that the proposed analytical approach was very accurate. The obtained analytical solution was in very good agreement with the numerical integration results, which proves the validity of our procedure. This indicates that the proposed approach is valid even if the non-linear differential equation does not contain any small or large parameters, which is a problem when applying some other methods. Compared to other analytical methods known in the literature, the proposed approach is very easy to use since it does not need higher-order approximations to obtain very good results, and it is very accurate when applied to complex problems.

Our procedure provides us with a simple way to optimally control and adjust the convergence of the solution, and can give good approximations in a few terms after only the first iteration. The convergence of the approximate solution obtained by the OAFM is determined by the optimal auxiliary functions $A_{1}$ and $A_{2}$, where the values of the convergence-control parameters $C_{i}, D_{j}$, and $E_{k}$ are optimally determined in a rigorous way.

The proposed dynamical model describing the generator wind turbine system under a simple wind gust allows for analysis of both mechanical and electrical phenomena and determining the performances of the dynamic regime produced by wind turbulence. It was proved that the wind speed can be considered in the system of equations describing the dynamic model to predict the system response to specific changes in speed. For this purpose, it is necessary to know the wind profile as a function of time. The present study is limited to only one type of wind profile, and in our future research work we intend to develop other approaches when modeling this profile describing the gust.

Finally, it is observed that the proposed approach allows an analytical solution to be obtained, which is very advantageous and useful for automatic control systems and protection systems used in this kind of aero-electric installation.

Concerning the proposed method as a whole, there are no known limitations to its application, and in our future works we will be concerned with applying this promising method to even more complicated strongly non-linear problems from real life.

Author Contributions: Conceptualization, N.H. and V.M.; Formal analysis, N.H. and V.M.; Funding acquisition, N.H. and F.D.; Investigation, N.H., V.M. and G.M.; Methodology, N.H., V.M. and G.M.; Project administration, N.H.; Resources, N.H.; Software, N.H.; Supervision, N.H.; Validation, N.H. and F.D.; Visualization, N.H.; Writing—original draft, N.H.; Writing—review \& editing, F.D.

Funding: This research received no external funding. 
Conflicts of Interest: The authors declare no conflict of interest.

\section{References}

1. Carlin, P.W.; Laxson, A.S.; Muljadi, E.B. The history and state of the art of variable-speed wind turbine technology. Wind Energy 2003, 6, 129-159. [CrossRef]

2. Herisanu, N.; Marinca, V.; Madescu, G. An analytical approach to non-linear dynamical model of a permanent magnet synchronous generator. Wind Energy 2015, 18, 1657-1670. [CrossRef]

3. Wasynczuk, O.; Man, D.T.; Sullivan, J.P. Dynamic behavior of a class of wind turbine generators during random wind fluctuation. IEEE Trans. Power Appl. Syst. 1981, 100, 2837-2845. [CrossRef]

4. Papathanassiou, S.A.; Papadopulos, M.P. Dynamic behaviour of variable speed wind turbines under stochastic wind. IEEE Trans. Energy Convers. 1999, 14, 1617-1623. [CrossRef]

5. Shariatpanah, H.; Fadeinedjad, R.; Rashidinejad, M. A new model for PMSG-based wind turbine with yaw control. IEEE Trans. Energy Convers. 2013, 28, 929-937. [CrossRef]

6. Nichita, C.; Luca, D.; Dakyo, B.; Ceanga, E. Large band simulation of the wind speed for real time wind turbine simulators. IEEE Trans. Energy Convers. 2002, 17, 523-529. [CrossRef]

7. Giaourakis, D.G.; Safacas, A.N. Quantitative and qualitative behavior analysis of a DFIG wind energy conversion system by a wind gust and converter faults. Wind Energy 2016, 19, 527-546. [CrossRef]

8. Borowy, B.S.; Salameh, Z.M. Dynamic response of a stand-alone wind energy conversion system with battery energy storage to a wind gust. IEEE Trans. Energy Convers. 1997, 12, 73-78. [CrossRef]

9. Bystryk, J.; Sullivan, P.E. Small wind turbine power control in intermittent wind gusts. J. Wind Eng. Ind. Aerodyn. 2011, 99, 624-637. [CrossRef]

10. Boldea, I. Variable Speed Generators; CRC Press: Boca Raton, FL, USA, 2006.

11. Vas, P. Electrical Machines and Drives. A Space-Vector Theory Approach; Claredon Press: Oxford, UK, 1996.

12. Binder, A. Elektrische Maschinen und Antriebe; Springer: Berlin/Heidelberg, Germany, 2012.

13. Ong, C.M. Dynamic Simulation of Electric Machinery; Prentice Hall: Upper Saddle River, NJ, USA, 1998.

14. Nayfeh, A.H. Introduction to Perturbation Techniques; Wiley: New York, NY, USA, 1993.

15. Awrejcewicz, J.; Krysko, V.A. Introduction to Asymptotic Methods; Chapman and Hall/CRC Press: Boca Raton, FL, USA, 2006.

16. Aksoy, Y.; Pakdemirli, M.; Abbasbandy, S.; Boyaci, H. New perturbation-iteration solutions for nonlinear heat transfer equations. Int. J. Numer. Methods Heat Fluid Flow 2012, 22, 814-828. [CrossRef]

17. Herisanu, N.; Marinca, V. An iteration procedure with application to Van der Pol oscillator. Int. J. Nonlinear Sci. Numer. Simul. 2009, 10, 353-361. [CrossRef]

18. Bildik, N.; Deniz, S. Comparative study between Optimal Homotopy Asymptotic Method and Perturbation-Iteration technique for different types of nonlinear equations. Iran. J. Sci. Technol. Trans. Sci. 2018, 42, 647-654. [CrossRef]

19. Abbasbandy, S.; Jalili, M. Determination of optimal convergence-control parameter value in homotopy analysis method. Numer. Algorithms 2013, 64, 593-605. [CrossRef]

20. Gupta, A.K.; Ray, S.S. Comparison between homotopy perturbation method and optimal homotopy asymptotic method for the soliton solutions of Boussinesq-Burger equations. Comput. Fluids 2014, 103, 34-41. [CrossRef]

21. Herisanu, N.; Marinca, V. Optimal homotopy perturbation method for a non-conservative dynamical system of a rotating electrical machine. Z. Nat. A 2012, 67, 509-516. [CrossRef]

22. Marinca, V.; Herisanu, N. The Optimal Homotopy Asymptotic Method. Engineering Applications; Springer: Cham, Switzerland, 2015.

23. Marinca, V.; Herisanu, N. On the flow of a Walters-type B viscoelastic fluid in a vertical channel with porous wall. Int. J. Heat Mass Transf. 2014, 79, 146-165. [CrossRef]

24. Rashidi, M.M.; Efrani, E. The modified differential transform method for investigating nano boundary-layers over stretching surfaces. Int. J. Numer. Methods Heat Fluid Flow 2011, 21, 864-883. [CrossRef]

25. Krause, P.C.; Wasynczuk, O.; Sudhoff, S.D. Analysis of Electrical Machinery; IEEE Press: New York, NY, USA, 1995.

26. Heier, S. Grid Integration of Wind Energy Conversion Systems; John Wiley \& Sons Ltd.: Chichester, UK, 1998. 
27. Anderson, P.M.; Bose, A. Stability simulation of wind turbine systems. IEEE Trans. Power Appl. Syst. 1983, 102, 3791-3795. [CrossRef]

28. Chatelain, J. Traite d'electricite. Machines Electriques; Presses Polytechniques Romandes: Lausanne, Switzerland, 1989.

29. Herisanu, N.; Marinca, V. Free oscillations of Euler-Bernoulli Beams on nonlinear Winkler-Pasternak foundation. In Acoustics and Vibration of Mechanical Structures-AVMS-2017; Springer: Cham, Switzerland, 2018; Volume 198, pp. 41-48.

(C) 2019 by the authors. Licensee MDPI, Basel, Switzerland. This article is an open access article distributed under the terms and conditions of the Creative Commons Attribution (CC BY) license (http:/ / creativecommons.org/licenses/by/4.0/). 